\title{
Conservative Dynamics of Binary Systems to Third Post-Minkowskian Order from the Effective Field Theory Approach
}

\author{
Gregor Kälin $\odot,{ }^{1}$ Zhengwen Liu $\odot,{ }^{2}$ and Rafael A. Porto $\odot^{2}$ \\ ${ }^{1}$ SLAC National Accelerator Laboratory, Stanford University, Stanford, California 94309, USA \\ ${ }^{2}$ Deutsches Elektronen-Synchrotron DESY, Notkestrasse 85, 22607 Hamburg, Germany
}

(Received 16 July 2020; accepted 1 December 2020; published 22 December 2020)

\begin{abstract}
We derive the conservative dynamics of nonspinning binaries to third post-Minkowskian order, using the effective field theory (EFT) approach introduced in [G. Kälin and R. A. Porto, J. High Energy Phys. 11 (2020) 106] together with the boundary-to-bound dictionary developed in [G. Kälin and R. A. Porto, J. High Energy Phys. 01 (2020) 072; J. High Energy Phys. 02 (2020) 120.]. The main ingredient is the scattering angle, which we compute to $\mathcal{O}\left(G^{3}\right)$ via Feynman diagrams. Adapting to the EFT framework powerful tools from the amplitudes program, we show how the associated (master) integrals are bootstrapped to all orders in velocities via differential equations. Remarkably, the boundary conditions can be reduced to the same integrals that appear in the EFT with post-Newtonian sources. For the sake of comparison, we reconstruct the Hamiltonian and the classical limit of the scattering amplitude. Our results are in perfect agreement with those in Bern et al. [Phys. Rev. Lett. 122, 201603 (2019); J. High Energy Phys. 10 (2019) 206].
\end{abstract}

DOI: 10.1103/PhysRevLett.125.261103

Introduction.-The discovery potential heralded by the new era of gravitational wave science $[1,2]$ has motivated high-accuracy theoretical predictions for the dynamics of binary systems [3-5]. This is particularly important for the inspiral phase of small relative velocities $(v / c \ll 1)$, covering a large portion of the cycles in the detectors' band for many events of interest, which is amenable to perturbative treatments like the celebrated post-Newtonian (PN) expansion [6,7]. Notably, in parallel with more "traditional" approaches in general relativity, e.g., Refs. [8-12], in recent years ideas from particle physics, such as effective field theories (EFTs) similar to those used to study bound states of strongly interacting particles [13-18], and modern tools from scattering amplitudes connecting gravity to YangMills theory and bypassing Feynman diagrams [19,20], have found their way into the classical two-body problem in gravity. Although more recent, these novel tools have made a key contribution to the knowledge of the conservative dynamics of binary systems, in both the PN regime as well as the post-Minkowskian (PM) expansion in powers of $G$ (Newton's constant), with the present state of the art reaching the fourth PN (4PN) [21-28] and third PM (3PM) [29-31] orders for nonspinning bodies, respectively. Partial results are also known to 5PN (static) [32,33] and

Published by the American Physical Society under the terms of the Creative Commons Attribution 4.0 International license. Further distribution of this work must maintain attribution to the author(s) and the published article's title, journal citation, and DOI. Funded by SCOAP.
$6 \mathrm{PN}[34,35]$; radiation and spin are incorporated in, e.g., Refs. [36-59].

Gravitational scattering amplitudes [29-31] find a natural habitat in the PM regime of a quantum world, which, at first, appears to bear little connection to the classical bound states where traditional PN tools [6] and the EFT approach [18] have been applied so far. While this can be circumvented by the universal character of the interaction, which is independent of the state, one still has to extract the classical part of the amplitude. In the framework of Refs. [29-31], this relies on the large angular momentum limit $\hbar / J \rightarrow 0$ (resulting also in a series of spurious infrared divergences removed by a matching computation). The procedure, however, was challenged in Ref. [60], with doubts (some addressed in Refs. [34,35]) on the validity of the 3PM Hamiltonian in Refs. [29,30]. In light of its relevance and demand for even higher accuracy [61], a systematic, scalable, and purely classical approach to observables in the PM regime was thus imperative.

Building upon the universal boundary-to-bound (B2B) dictionary, relating scattering data directly to gauge-invariant observables for generic orbits through analytic continuation $[62,63]$, a novel PM framework was developed in Ref. [64] using the EFT machinery and readily implemented for bound states to $\mathcal{O}\left(G^{2}\right)$. (See, e.g., Refs. [60,61,65-68] for alternative routes.) In this Letter, we report the next step in the EFT approach, namely, the computation of the conservative binary dynamics to 3PM order. This entails the calculation of the scattering angle to next-to-next-to-leading order (NNLO) in $G$ via Feynman diagrams. Remarkably, we find that the associated (master) 
integrals can be bootstrapped from their PN counterparts through differential equations in the velocity [69], as advocated in Ref. [70], paving the way forward to higher-order computations. For the sake of comparison, we reconstruct the Hamiltonian as well as the (infraredfinite) amplitude in the classical limit and find complete agreement with the results in Refs. [29,30]. Our derivation thus independently confirms the connection between the amplitude and the center-of-mass (c.m.) momentum (impetus formula) [62] and the legitimacy of the program to extract classical physics from scattering amplitudes [29-31,55-59,62,63,70-96]. At the same time, we explicitly demonstrate the power of the EFT and B2B framework [62-64], which by design can be systematized to all orders.

The EFT framework.-The starting point is the effective action from which we derive the scattering trajectories. We proceed by integrating out the metric field $g_{\mu \nu}=\eta_{\mu \nu}+$ $h_{\mu \nu} / M_{\mathrm{Pl}}\left(\right.$ with $\left.M_{\mathrm{Pl}}^{-1} \equiv \sqrt{32 \pi G}\right)$ :

$$
e^{i S_{\mathrm{eff}}}=\int \mathcal{D} h_{\mu \nu} e^{i S_{\mathrm{EH}}[h]+i S_{\mathrm{GF}}[h]+i S_{\mathrm{pp}}\left[x_{a}, h\right]},
$$

in the (classical) saddle-point and weak-field approximations. We work with the Einstein-Hilbert action $S_{\mathrm{EH}}$ and the convention $\eta_{\mu \nu}=\operatorname{diag}(+,-,-,-)$. The gauge fixing $S_{\mathrm{GF}}$ is adjusted to simplify the Feynman rules [64]. We use the (Polyakov) point-particle effective action:

$$
S_{\mathrm{pp}}=-\sum_{a=1,2} \frac{m_{a}}{2} \int d \tau_{a} g_{\mu \nu}\left(x_{a}^{\alpha}\right) v_{a}^{\mu} v_{a}^{\nu}+\cdots,
$$

with $\tau_{a}$ the proper time. The ellipses include higherderivative terms accounting for finite-size effects and counterterms to remove (classical) ultraviolet divergences $[13,64]$. As usual, we use dimensional regularization.

Impulse from action.-From the action, we read off the effective Lagrangian at each order in $G: \mathcal{L}_{\text {eff }}=\mathcal{L}_{0}+\mathcal{L}_{1}+$ $\mathcal{L}_{2}+\mathcal{L}_{3}+\cdots$. Although it may be nonlocal in time when radiation-reaction effects are included [8,24], it is manifestly local with only potential modes [64]. Using the effective Lagrangian, we obtain the trajectories

$$
x_{a}^{\mu}\left(\tau_{a}\right)=b_{a}^{\mu}+u_{a}^{\mu} \tau_{a}+\sum_{n} \delta^{(n)} x_{a}^{\mu}\left(\tau_{a}\right),
$$

with $u_{a}^{\mu}$ the velocity at infinity, obeying $u_{a}^{2}=1$, and $b^{\mu} \equiv$ $b_{1}^{\mu}-b_{2}^{\mu}$ the impact parameter. For instance, at LO,

$$
\begin{aligned}
\delta^{(1)} x_{1}^{\mu}\left(\tau_{1}\right)= & -\frac{m_{2}}{8 M_{\mathrm{Pl}}^{2}}\left[\left(2 \gamma^{2}-1\right) \eta^{\mu \nu}-2\left(2 \gamma u_{2}^{\mu}-u_{1}^{\mu}\right) u_{1}^{\nu}\right] \\
& \times \int_{k} \frac{i k_{\nu} \hat{\delta}\left(k \cdot u_{2}\right) e^{i k \cdot b}}{k^{2}\left(k \cdot u_{1}-i 0^{+}\right)^{2}} e^{i\left(k \cdot u_{1}-i 0^{+}\right) \tau_{1}} .
\end{aligned}
$$

We use the notation $\int_{k} \equiv \int\left[d^{4} k /(2 \pi)^{4}\right], \hat{\delta}(x) \equiv 2 \pi \delta(x)$, and

$$
\gamma \equiv u_{1} \cdot u_{2}=\frac{p_{1} \cdot p_{2}}{m_{1} m_{2}}=\frac{E_{1} E_{2}+\boldsymbol{p}^{2}}{m_{1} m_{2}},
$$

where $E_{a}=\sqrt{\boldsymbol{p}^{2}+m_{a}^{2}}$ and $\pm \boldsymbol{p}$ is the incoming c.m. momentum. Notice the factor of $\left(k \cdot u_{1}-i 0^{+}\right)^{-1}$, with the $i 0^{+}$to ensure convergence of the time integrals, which resembles the linear propagators appearing in heavy-quark effective theory [97]. The pole shifts to $\left(k \cdot u_{2}+i 0^{+}\right)^{-1}$ for particle 2 . The impulse follows from the effective action:

$$
\Delta p_{a}^{\mu}=-\eta^{\mu \nu} \int_{-\infty}^{+\infty} d \tau_{a} \frac{\partial \mathcal{L}_{\mathrm{eff}}}{\partial x_{a}^{\nu}}\left[x_{a}\left(\tau_{a}\right)\right],
$$

where the overall sign is due to our conventions. The impulse can then be solved iteratively, starting with the undeflected trajectory in Eq. (3). Notice that all of the $\mathcal{L}_{k<n}$ 's contribute to $n \mathrm{PM}$ order and must be evaluated on the trajectories up to $(n-k)$ th order in $G$. We refer to this procedure as iterations [64]. The scattering angle

$$
\frac{\chi}{2}=\sum_{n} \chi_{b}^{(n)}\left(\frac{G M}{b}\right)^{n}=\sum_{n} \frac{\chi_{j}^{(n)}}{j^{n}},
$$

with $1 / j=G M \mu /\left(p_{\infty} b\right)$, is obtained from the relation

$$
2 \sin \frac{\chi}{2}=2\left[\frac{\chi}{2}-\frac{1}{6}\left(\frac{\chi}{2}\right)^{3}+\cdots\right]=\frac{\sqrt{-\Delta p_{a}^{2}}}{p_{\infty}},
$$

where

$p_{\infty}=\mu \frac{\sqrt{\gamma^{2}-1}}{\Gamma}, \quad \Gamma \equiv \frac{E}{M}=\sqrt{1+2 \nu(\gamma-1)}$,

with $E$ and $M$ the total mass and energy, respectively. We use the notation $\mu=m_{1} m_{2} / M$ for the reduced mass and $\nu=\mu / M$ for the symmetric mass ratio.

The impulse may be further split into a contribution along the direction of the impact parameter as well as a term proportional to the velocities [64]. Because of momentum conservation and the on-shell condition, we have

$$
\left(p_{a}+\Delta p_{a}\right)^{2}=p_{a}^{2} \Rightarrow 2 p_{a} \cdot \Delta p_{a}=-\Delta p_{a}^{2} .
$$

Moreover, since $\Delta^{(1)} p_{1}^{\mu} \propto b^{\mu}$ at leading PM order [64] and $b \cdot u_{a}=0$, we can use Eq. (10) to solve iteratively for the component along the velocities. This allows us to restrict the derivation of the impulse to the perpendicular plane [64].

Feynman integrals.-To 3PM order, the Feynman topologies are shown in Fig. 1. The computation yields fourdimensional relativistic integrals constrained by a series of $\delta$ functions, $\delta\left(k_{i} \cdot u_{a}\right)$, which arise due to the time integration in Eq. (6) after inputting Eq. (3). Moreover, in addition to the standard factors of $1 / k^{2}$ from the gravitational field, we have linear propagators, as in Eq. (4), which are needed to compute the iterations. As we mentioned, we restrict ourselves to the computation of the impulse in the direction of the impact parameter. The derivation is then reduced to a series of terms proportional to the Fourier transform in the "transfer momentum":

$$
\int_{q} \hat{\delta}\left(q \cdot u_{1}\right) \hat{\delta}\left(q \cdot u_{2}\right) i q^{\mu} t^{s} M_{n_{1} n_{2} ; i_{1} \ldots i_{5}}^{(a, \tilde{a})}(q, \gamma) e^{i q \cdot b}
$$


where the factor of $t^{s}$, with $t \equiv-q^{2}$, depends on the tensor reduction of the given diagram. We find the following (cut) "two-loop" integrals [98]:

$$
M_{n_{1} n_{2} ; i_{1} \ldots i_{5}}^{(a, \tilde{a})}(q, \gamma) \equiv \int_{k_{1}, k_{2}} \frac{\hat{\delta}\left(k_{1} \cdot u_{a}\right) \hat{\delta}\left(k_{2} \cdot u_{\tilde{a}}\right)}{A_{1, \phi^{2}}^{n_{1}} A_{2, \tilde{\phi}}^{n_{2}} D_{1}^{i_{1}} \ldots D_{5}^{i_{5}}}
$$

are sufficient to $3 \mathrm{PM}$ order, where $(\not=2, \not=1)$

$$
\begin{aligned}
A_{1, \phi} & =k_{1} \cdot u_{\phi}, \quad A_{2, \tilde{\phi}}=k_{2} \cdot u_{\tilde{\phi}}, \quad D_{1}=k_{1}^{2}, \quad D_{2}=k_{2}^{2}, \\
D_{3} & =\left(k_{1}+k_{2}-q\right)^{2}, \quad D_{4}=\left(k_{1}-q\right)^{2}, \quad D_{5}=\left(k_{2}-q\right)^{2} .
\end{aligned}
$$

All the integrals we encounter in our computation, including the iterations, can be embedded into the family in Eq. (12) with different choices of $(a, \tilde{a})$. The $i 0$ prescription is such the $u_{1,2}$ are always accompanied by $\mp i 0^{+}$, as in Eq. (4). The other cases are obtained by different symmetrizations [98]. We keep only nonanalytic terms in $t$ which yield long-range interactions [64]. We outline the integration procedure momentarily. The outcome is the scaling

$$
t^{s} M_{n_{1} n_{2} ; i_{1} \ldots i_{5}}^{(a, \tilde{a})} \propto \frac{1}{\epsilon} t^{-2 \epsilon},
$$

with $\epsilon=(4-D) / 2$, which gives for the impulse in Eq. (11) the expected $b^{\mu} / b^{4}$ in $D=4$. The poles (and $\log \bar{\mu}$ 's) in dimensional regularization accompanying the $\log t$ 's produce contact terms that neatly drop out without referring to subtraction schemes [64].

Potential modes. - In the framework of the PN expansion, the integrals would be performed using a mode factorization into potential $\left(k_{0} \ll|\boldsymbol{k}|\right)$ and radiation $\left(k_{0} \sim\right.$ $|\boldsymbol{k}|)$ modes while keeping manifest power counting in the velocity $[13,18,99]$. The computation with potential modes then reduces to a series of three-dimensional (massless) integrals. In contrast, in the PM scheme we ought to keep the propagators fully relativistic. The associated Feynman integral still receive contributions from both potential and radiation modes (yielding real and imaginary parts). We are interested here in the conservative sector, and we ignore for now radiation-reaction effects. [Hereditary tail effects, which enter in the conservative dynamics through nonlocal contributions to the effective action, e.g., [8,24], first appear at $\mathcal{O}\left(G^{2} a^{2} v^{2}\right) \sim \mathcal{O}\left(G^{4} v^{2}\right)$ [28], namely, 4PM.] As discussed in Ref. [64], to isolate the potential modes, we adapt to our EFT framework the powerful tools developed in Refs. [29,30,70]. Notably, we make use of the methodology of differential equations using boundary conditions from the (static) limit $\gamma \rightarrow 1$ [70].

On the one hand, for Figs. 1(c) and 1(d), only the $M_{n_{1}, n_{2} ; \ldots}^{(1,1)}$ in Eq. (12) are needed, with $\left(n_{1}, n_{2}\right) \leq 0$, plus mirror images. These integrals, which contribute to the onepoint function of a (boosted) Schwarzschild background, can be computed in the rest frame:

$$
u_{1}=(1,0,0,0), \quad u_{2}=(\gamma, \gamma \beta, 0,0),
$$

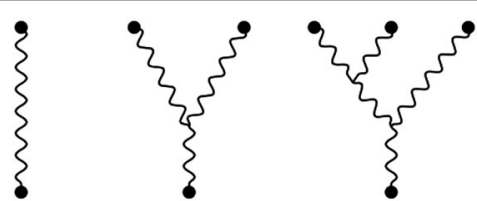

(a) (b)

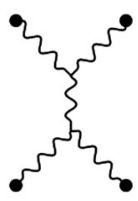

(e)

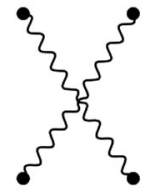

(f) (c)



(g)
FIG. 1. Feynman topologies to 3PM [64].

with $\beta \gamma=\sqrt{\gamma^{2}-1}$ [64]. At the end of the day, they turn into the same type that appear in the static limit of the PN expansion; see, e.g., Ref. [21]. For Figs. 1(e)-1(g), on the other hand, the $M_{n_{1} n_{2} ; \ldots}^{(1,2)}$ are required instead, also with $\left(n_{1}, n_{2}\right) \leq 0$. Remarkably, the associated integrals for all these diagrams can be decomposed into a basis involving only the $M_{00 ; \ldots}^{(1,2)}$ subset [98]. Furthermore, using integration by part (IBP) relationships [100,101], the contribution from Figs. 1(e) and 1(f) reduces to integrals with $i_{3}=0$. It is then straightforward to show that both diagrams vanish in $D=4$. (This is reminiscent of the fact that they do not enter at 2PN either [21].) Using the IBP relations and the aid of FIRE6 [102] and LiteRed [103], as well as symmetry arguments, the calculation of the remaining (so-called $H$ ) diagram in Fig. 1(g) is reduced to the following basis [98]:

$$
\left\{I_{11111}, I_{11211}, I_{01101}, I_{11011}, I_{00211}, I_{00112}, I_{00111}\right\},
$$

with $I_{i_{1} \ldots i_{5}} \equiv M_{00 ; i_{1} \ldots i_{5}}^{(1,2)}$. For the computation, we follow Ref. [104] and various tools, e.g., epsilon [105], to construct a canonical basis $\vec{h}=\left\{h_{n=1 \ldots 7}\right\}$ such that the velocity dependence is obtained via differential equations:

$$
\partial_{x} \vec{h}(x, \epsilon)=\epsilon \mathbb{M}(x) \vec{h}(x, \epsilon)
$$

with $\gamma=\left(x^{2}+1\right) /(2 x)$, as advocated in Ref. [70]. Because the set in Eq. (16) contains up to five (quadratic) propagators only, the associated boundary conditions in our case are then reduced to the same type of integrals that appear in the PN regime at two loops (kite diagrams; e.g., Ref. [25]). It turns out that only a handful contribute to the $H$ diagram in $D=4$, featuring the much anticipated factor of $\log x$ observed in Refs. [29,30,70].

To complete the derivation, we have to include the iterations. Surprisingly, the set in Eq. (16) is (almost) sufficient for all the contributions. For instance, iterations involving the deflection due to Fig. 1(a) at LO order for the impulse due to Fig. 1(b), and vice versa, follow from Eq. (16). Yet, for the deflection from Fig. 1(a) to NLO, 
additional integrals are needed, resembling other (cut) topologies in Refs. [30,70]. In our case, we need the following two:

$$
\left\{M_{11 ; 11100}^{(1,1)}, M_{11 ; 11100}^{(1,2)}\right\} .
$$

In principle, we find all $\pm i 0$ combinations. Naively, due to the lack of "crossing" (e.g., $u_{1} \rightarrow-u_{1}$ ) in the potential region, the connection between them is not obvious; see Ref. [70]. Yet, we can show that these integrals are related in the static limit (see below). The upshot is that various $\pm i 0$ choices differ by relative factors of 2 . These turn out to be crucial to ensure the cancellation of intermediate spurious infrared poles $\propto t^{-2 \epsilon} / \epsilon^{2}$ [98]. Because of the presence of divergences, however, their computation is somewhat subtle. For the first one, we can readily go to the rest frame in Eq. (15) producing a $D-1$ integral. We then use the symmetrization described in Ref. [70]. Alternatively, it may be computed using the prescription in Refs. [29-31] in the $u_{2}$ frame. Both can be adapted to all $\pm i 0$ choices. The result is proportional to (twice) the standard one-loop bubble integrals with static PN sources [21], although in $D-2$ dimensions. The same trick does not apply to the latter, but it can be easily incorporated into the canonical basis to obtain its $\gamma$ dependence. Yet, due to a divergence in the static limit, we need some care with the boundary condition. This is accounted for in the canonical basis by pulling out the relevant factor of $\beta$ (and $\epsilon$ ). Once again, we perform the integral in the rest frame, expand in small velocity, and retain the leading term in $1 / \beta$. In this limit, the $M_{11 ; \ldots}^{(1,2)}$ integral turns out to be equivalent (modulo different $\pm i 0$ choices) to the $M_{11 ; \ldots}^{(1,1)}$ counterpart. We have checked all these relationships explicitly via a standard $\alpha$ parameterization [106]. At the end, as expected, the associated divergences cancel out in the final answer without subtractions.

The above steps culminate in the derivation of the master integrals in the potential region via differential equations. Using various arguments, the boundary conditions are reduced to the master integrals that appear in the static limit of the PN expansion at the same loop order. See Ref. [98] for a more detailed discussion.

Scattering data.-The result for the impulse now follows from basic algebraic manipulations, and we arrive at

$$
\begin{aligned}
\Delta^{(3)} p_{1}^{\mu}= & \frac{G^{3} b^{\mu}}{\left|b^{2}\right|^{2}}\left(\frac{16 m_{1}^{2} m_{2}^{2}\left(4 \gamma^{4}-12 \gamma^{2}-3\right) \sin h^{-1} \sqrt{\frac{\gamma-1}{2}}}{\left(\gamma^{2}-1\right)}\right. \\
& -\frac{4 m_{1}^{2} m_{2}^{2} \gamma\left(20 \gamma^{6}-90 \gamma^{4}+120 \gamma^{2}-53\right)}{3\left(\gamma^{2}-1\right)^{5 / 2}} \\
& \left.-\frac{2 m_{1} m_{2}\left(m_{1}^{2}+m_{2}^{2}\right)\left(16 \gamma^{6}-32 \gamma^{4}+16 \gamma^{2}-1\right)}{\left(\gamma^{2}-1\right)^{5 / 2}}\right) \\
& +\frac{3 \pi}{2} \frac{\left(2 \gamma^{2}-1\right)\left(5 \gamma^{2}-1\right)}{\left(\gamma^{2}-1\right)^{2}} \frac{G^{3} M^{2} \mu}{\left|b^{2}\right|^{3 / 2}} \\
& \times\left[\left(\gamma m_{2}+m_{1}\right) u_{2}^{\mu}-\left(\gamma m_{1}+m_{2}\right) u_{1}^{\mu}\right] .
\end{aligned}
$$

The last term, which does not feature in the deflection angle at this order, is obtained from Eq. (10) and the result in Ref. [64]. Hence, using Eq. (8), the 1PM angle (cube), and the 2PM impulse along the velocities in Ref. [64], we find

$$
\begin{aligned}
\frac{\chi_{b}^{(3)}}{\Gamma}= & \frac{1}{\left(\gamma^{2}-1\right)^{3 / 2}}\left[-\frac{4 \nu}{3} \gamma \sqrt{\gamma^{2}-1}\left(14 \gamma^{2}+25\right)\right. \\
& +\frac{\left(64 \gamma^{6}-120 \gamma^{4}+60 \gamma^{2}-5\right)[1+2 \nu(\gamma-1)]}{3\left(\gamma^{2}-1\right)^{3 / 2}} \\
& \left.-8 \nu\left(4 \gamma^{4}-12 \gamma^{2}-3\right) \sin h^{-1} \sqrt{\frac{\gamma-1}{2}}\right],
\end{aligned}
$$

which, using $\chi_{j}^{(3)}=\left(p_{\infty} / \mu\right)^{3} \chi_{b}^{(3)}=\left(\sqrt{\gamma^{2}-1} / \Gamma\right)^{3} \chi_{b}^{(3)}$, is in agreement with the derivation in Refs. [29,30]; see also Ref. [61].

B2B map.-The scattering data allow us to construct the (reduced) radial action $[62,63]$

$$
i_{r}=\frac{p_{\infty}}{\sqrt{-p_{\infty}^{2}}} \chi_{j}^{(1)}-j\left(1+\frac{2}{\pi} \sum_{n=1}^{\infty} \frac{\chi_{j}^{(2 n)}}{(1-2 n) j^{2 n}}\right),
$$

via analytic continuation to $\gamma<1$. As we discussed in Refs. $[62,63]$, the natural power counting in $1 / j$ in the PM expansion requires the (so far unknown) $\chi_{j}^{(4)}$ coefficient. The latter can be written, using the results in Refs. $[62,63]$, as

$$
\chi_{j}^{(4)}=\frac{3 \pi}{8 M^{4} \mu^{4}}\left(P_{1} P_{3}+\frac{1}{2} P_{2}^{2}+p_{\infty}^{2} P_{4}\right),
$$

with the $P_{n}$ 's from the expansion of the c.m. momentum

$$
p^{2}=p_{\infty}^{2}+\sum_{n=1}^{\infty} P_{n}(E)\left(\frac{G}{r}\right)^{n} .
$$

The $P_{n}$ 's can also be obtained from the scattering angle, as described in Refs. [62,63]. For instance, inverting the relation

$$
\chi_{j}^{(3)}=\frac{1}{M^{3} \mu^{3} p_{\infty}^{3}}\left(-\frac{P_{1}^{3}}{24}+p_{\infty}^{2} \frac{P_{1} P_{2}}{2}+p_{\infty}^{4} P_{3}\right),
$$

together with Eq. (20) and the results in Ref. [64], yields

$$
\begin{aligned}
\frac{P_{3}}{M^{3} \mu^{2}}= & {\left[\frac{18 \gamma^{2}-1}{2 \Gamma}+\frac{8 \nu}{\Gamma}\left(3+12 \gamma^{2}-4 \gamma^{4}\right) \frac{\sin h^{-1} \sqrt{\frac{\gamma-1}{2}}}{\sqrt{\gamma^{2}-1}}\right.} \\
& +\frac{\nu}{6 \Gamma}\left(6-206 \gamma-108 \gamma^{2}-4 \gamma^{3}\right. \\
& \left.\left.+\frac{18 \Gamma\left(1-2 \gamma^{2}\right)\left(1-5 \gamma^{2}\right)}{(1+\Gamma)(1+\gamma)}\right)\right] .
\end{aligned}
$$

This compact expression encodes all the information at 3PM order. It can be analytically continued to negative binding energies $(\gamma<1)$ to derive observables for binary systems via the B2B map. Because of the factor of $p_{\infty}^{2}$ in Eq. (22), and since Eq. (23) has a well-defined static limit, the contribution in Eq. (21) from $P_{4}$ is subleading in the PN expansion. This 
allows us to perform a consistent PN truncation by keeping the $P_{n \leq 3}$ terms in Eq. (22) (ignoring also higher orders in $1 / j$ which are PN suppressed). This is carried out in detail in Refs. $[62,63]$ and shown to agree with the literature in the overlapping regime of validity.

Amplitude and Hamiltonian.- It is instructive to use the B2B dictionary to also reconstruct both the classical limit of the scattering amplitude as well as the Hamiltonian for the two-body system in the c.m. (isotropic) frame. Using the relationship found in Ref. [62],

$$
\boldsymbol{p}^{2}=p_{\infty}^{2}+\frac{1}{2 E} \int d^{3} \boldsymbol{r} \mathcal{M}\left(p_{\infty}, \boldsymbol{q}\right) e^{i \boldsymbol{q} \cdot \boldsymbol{r}},
$$

we immediately read off from Eq. (25) the (infrared-finite part of the) scattering amplitude in the classical limit, which agrees with the result in Ref. [30] [see Eq. (9.3)]. For the PM expansion of the Hamiltonian,

$$
H\left(r, \boldsymbol{p}^{2}\right)=\sum_{i} \frac{c_{i}\left(\boldsymbol{p}^{2}\right)}{i !}\left(\frac{G}{r}\right)^{i},
$$

the coefficients can also be expressed iteratively in terms of the $P_{n}$ 's in Eq. (23) [62]. To 3PM order, we find

$$
\begin{aligned}
\frac{c_{3}(\boldsymbol{p})}{3 !}= & -\frac{P_{3}(E)}{2 E \xi}+\frac{(3 \xi-1) P_{2}(E) P_{1}(E)}{4 E^{3} \xi^{3}} \\
& +\frac{\left[P_{2}(E) P_{1}^{\prime}(E)+P_{2}^{\prime}(E) P_{1}(E)\right]}{4 E^{2} \xi^{2}} \\
& -\frac{\left(5 \xi^{2}-5 \xi+1\right) P_{1}^{3}(E)}{16 E^{5} \xi^{5}}-\frac{(9 \xi-3) P_{1}^{2}(E) P_{1}^{\prime}(E)}{16 E^{4} \xi^{4}} \\
& -\frac{P_{1}^{2}(E) P_{1}^{\prime \prime}(E)}{16 E^{3} \xi^{3}}-\frac{P_{1}(E)\left[P_{1}^{\prime}(E)\right]^{2}}{8 E^{3} \xi^{3}},
\end{aligned}
$$

where a prime denotes a derivative with respect to $E$ and $\xi \equiv E_{1} E_{2} /\left(E_{1}+E_{2}\right)^{2}$. Inputting Eq. (25) and $P_{1,2}$ from the 2PM results [64], we exactly reproduce the $c_{3}$ in Refs. [29,30]. Notice, however, that the relevant PM information to compute observables through the B2B map is (more succinctly) encoded in Eq. (25) at two loops and, ultimately, the (yet to be computed) scattering angle at 4PM order.

Conclusions.-Using the EFT approach and B2B dictionary [62-64], we derived the conservative dynamics for nonspinning binary systems to 3PM order. Our results, purely within the classical realm, are in perfect agreement with those reported in Refs. [29,30], thus removing the objections raised in Ref. [60] against their validity. Even though, unlike the approach in Refs. [29,30], our derivation entails the use of a Feynman diagram, because of the simplifications of the EFT and B2B framework, just a handful are required (two of which are zero) at this order; see Fig. 1. Moreover, only massless integrals appear, and, as was already illustrated in Ref. [64], we do not encounter the (superclassical) infrared singularities which have, thus far, polluted the extraction of classical physics from the amplitudes program. By adapting to our EFT approach the methods in Refs. [29-31,70], we found that the contribution from potential modes to the master integrals can be computed to all orders in velocities using differential equations (without the need of the PN-type resummations in Refs. [29,30]). Remarkably, the boundary conditions are obtained from the knowledge of the same master integrals which appear in the static limit with PN sources to two loops, albeit in $D-1$ and $D-2$ dimensions. This implies that the PM dynamics can be bootstrapped from PN information (at least to NNLO). This is not surprising for the evaluation on the unperturbed trajectory, which serves as a stationary limit of the PM regime, but strikingly the same occurs for the iterations. Since master integrals for the PN expansion are known to four loops [25], bootstrapping integrals through differential equations could potentially give us up to the 5PM order.

We note also that the infusion of data from outside of PN and PM schemes can further simplify the computation. For instance, the test-particle limit in a Schwarzschild background provides us the value of the $M^{(1,1)}$ master integrals in the iterations. In turn, these are related to the $M^{(1,2)}$ family in the static limit. This would then allow us to read off their boundary condition directly from the test-body limit and, subsequently, the entire velocity dependence with the differential equations. The fact that we get extra mileage from the probe limit is not surprising [62]. What is remarkable, and more so due to the lack of crossing symmetry (while the spurious infrared poles from the master integrals ultimately cancel out, crossing may be restored by implementing the zero-bin subtraction to remove the overlap with other "soft" regions, as with potential and radiation modes in the PN case $[26,107])$, is the connection to $\mathcal{O}(\nu)$ corrections through the static limit and differential equations. Likewise, information from the gravitational self-force program [108,109] may be also used to aid the calculation in the PM expansion, e.g., $[35,60,61,65-67,110-113]$. Irrespectively of the weapon of choice, the B2B dictionary $[62,63]$ is imploring us to continue to even higher orders. The derivation of the needed 4PM scattering angle is ongoing in the EFT approach, which we have demonstrated here is a powerful framework, not only for PN calculations [13-18], but also in the PM regime $[64,114]$.

We thank Babis Anastasiou, Zvi Bern, Clifford Cheung, Lance Dixon, Claude Duhr, Julio Parra-Martinez, Radu Roiban, Chia-Hsien Shen, Mikhail Solon, Gang Yang, and Mao Zeng for useful discussions. We are grateful to Julio Parra-Martinez and Mao Zeng for helpful comments on the integration in the potential region. R. A.P. acknowledges financial support from the ERC Consolidator Grant "Precision Gravity: From the LHC to LISA" provided by the European Research Council (ERC) under the European Union's H2020 research and innovation program (Grant Agreement No. 817791). Z. L. and R. A. P. are also supported by the Deutsche Forschungsgemeinschaft (DFG) under Germany's Excellence Strategy (EXC 2121) "Quantum Universe" (390833306). G. K. is supported by 
the Knut and Alice Wallenberg Foundation under Grant No. KAW 2018.0441 and in part by the United States Department of Energy under Contract No. DE-AC0276 SF00515.

[1] B. P. Abbott et al. (LIGO Scientific and Virgo Collaborations), Phys. Rev. X 9, 031040 (2019).

[2] R. Abbott et al. (LIGO Scientific and Virgo Collaborations), arXiv:1912.11716.

[3] A. Buonanno and B. Sathyaprakash, arXiv:1410.7832.

[4] R. A. Porto, Fortschr. Phys. 64, 723 (2016).

[5] R. A. Porto, arXiv:1703.06440.

[6] L. Blanchet, Living Rev. Relativity 17, 2 (2014).

[7] G. Schäfer and P. Jaranowski, Living Rev. Relativity 21, 7 (2018).

[8] T. Damour, P. Jaranowski, and G. Schäfer, Phys. Rev. D 89, 064058 (2014).

[9] P. Jaranowski and G. Schäfer, Phys. Rev. D 92, 124043 (2015).

[10] L. Bernard, L. Blanchet, A. Bohe, G. Faye, and S. Marsat, Phys. Rev. D 93, 084037 (2016).

[11] L. Bernard, L. Blanchet, A. Bohe, G. Faye, and S. Marsat, Phys. Rev. D 96, 104043 (2017).

[12] T. Marchand, L. Bernard, L. Blanchet, and G. Faye, Phys. Rev. D 97, 044023 (2018).

[13] W. D. Goldberger and I. Z. Rothstein, Phys. Rev. D 73, 104029 (2006).

[14] W. D. Goldberger, Les Houches 86, 351 (2007).

[15] S. Foffa and R. Sturani, Classical Quantum Gravity 31, 043001 (2014).

[16] I. Rothstein, Gen. Relativ. Gravit. 46, 1726 (2014).

[17] V. Cardoso and R. A. Porto, Gen. Relativ. Gravit. 46, 1682 (2014).

[18] R. A. Porto, Phys. Rep. 633, 1 (2016).

[19] H. Elvang and Y.-t. Huang, Scattering Amplitudes in Gauge Theory and Gravity (Cambridge University Press, Cambridge, England, 2015).

[20] Z. Bern, J. J. Carrasco, M. Chiodaroli, H. Johansson, and R. Roiban, arXiv:1909.01358.

[21] J. B. Gilmore and A. Ross, Phys. Rev. D 78, 124021 (2008).

[22] S. Foffa and R. Sturani, Phys. Rev. D 84, 044031 (2011).

[23] S. Foffa and R. Sturani, Phys. Rev. D 87, 064011 (2013).

[24] C. R. Galley, A. K. Leibovich, R. A. Porto, and A. Ross, Phys. Rev. D 93, 124010 (2016).

[25] S. Foffa, P. Mastrolia, R. Sturani, and C. Sturm, Phys. Rev. D 95, 104009 (2017).

[26] R. A. Porto and I. Z. Rothstein, Phys. Rev. D 96, 024062 (2017).

[27] S. Foffa and R. Sturani, Phys. Rev. D 100, 024047 (2019).

[28] S. Foffa, R. A. Porto, I. Rothstein, and R. Sturani, Phys. Rev. D 100, 024048 (2019).

[29] Z. Bern, C. Cheung, R. Roiban, C.-H. Shen, M. P. Solon, and M. Zeng, Phys. Rev. Lett. 122, 201603 (2019).

[30] Z. Bern, C. Cheung, R. Roiban, C.-H. Shen, M. P. Solon, and M. Zeng, J. High Energy Phys. 10 (2019) 206.

[31] C. Cheung, I. Z. Rothstein, and M. P. Solon, Phys. Rev. Lett. 121, 251101 (2018).
[32] S. Foffa, P. Mastrolia, R. Sturani, C. Sturm, and W. J. Torres Bobadilla, Phys. Rev. Lett. 122, 241605 (2019).

[33] J. Blümlein, A. Maier, and P. Marquard, Phys. Lett. B 800, 135100 (2020).

[34] J. Blümlein, A. Maier, P. Marquard, and G. Schäfer, Phys. Lett. B 807, 135496 (2020).

[35] D. Bini, T. Damour, and A. Geralico, Phys. Rev. D 102, 024061 (2020).

[36] W. D. Goldberger and I. Z. Rothstein, Phys. Rev. D 73, 104030 (2006).

[37] W. D. Goldberger and A. Ross, Phys. Rev. D 81, 124015 (2010).

[38] A. Ross, Phys. Rev. D 85, 125033 (2012).

[39] C. R. Galley and A. K. Leibovich, Phys. Rev. D 86, 044029 (2012).

[40] A. K. Leibovich, N. T. Maia, I. Z. Rothstein, and Z. Yang, Phys. Rev. D 101, 084058 (2020).

[41] R. A. Porto, Phys. Rev. D 73, 104031 (2006).

[42] R. A. Porto and I. Z. Rothstein, Phys. Rev. Lett. 97, 021101 (2006).

[43] R. A. Porto and I. Z. Rothstein, arXiv:0712.2032.

[44] R. A. Porto, Phys. Rev. D 77, 064026 (2008).

[45] R. A. Porto and I. Z. Rothstein, Phys. Rev. D 78, 044012 (2008).

[46] R. A. Porto and I. Z. Rothstein, Phys. Rev. D 78, 044013 (2008).

[47] R. A. Porto, Classical Quantum Gravity 27, 205001 (2010).

[48] R. A. Porto, A. Ross, and I. Z. Rothstein, J. Cosmol. Astropart. Phys. 03 (2011) 009.

[49] R. A. Porto, A. Ross, and I. Z. Rothstein, J. Cosmol. Astropart. Phys. 09 (2012) 028.

[50] N. T. Maia, C. R. Galley, A. K. Leibovich, and R. A. Porto, Phys. Rev. D 96, 084064 (2017).

[51] N. T. Maia, C. R. Galley, A. K. Leibovich, and R. A. Porto, Phys. Rev. D 96, 084065 (2017).

[52] M. Levi and J. Steinhoff, arXiv:1607.04252.

[53] M. Levi, A. J. Mcleod, and M. Von Hippel, arXiv: 2003.02827.

[54] M. Levi, A. J. Mcleod, and M. Von Hippel, arXiv: 2003.07890.

[55] V. Vaidya, Phys. Rev. D 91, 024017 (2015).

[56] A. Guevara, A. Ochirov, and J. Vines, Phys. Rev. D 100, 104024 (2019).

[57] N. Arkani-Hamed, Y.-t. Huang, and D. O'Connell, J. High Energy Phys. 01 (2020) 046.

[58] M.-Z. Chung, Y.-t. Huang, J.-W. Kim, and S. Lee, J. High Energy Phys. 05 (2020) 105.

[59] Z. Bern, A. Luna, R. Roiban, C.-H. Shen, and M. Zeng, arXiv:2005.03071.

[60] T. Damour, Phys. Rev. D 102, 024060 (2020).

[61] A. Antonelli, A. Buonanno, J. Steinhoff, M. van de Meent, and J. Vines, Phys. Rev. D 99, 104004 (2019).

[62] G. Kälin and R. A. Porto, J. High Energy Phys. 01 (2020) 072.

[63] G. Kälin and R. A. Porto, J. High Energy Phys. 02 (2020) 120.

[64] G. Kälin and R. A. Porto, J. High Energy Phys. 11 (2020) 106. 
[65] T. Damour, Phys. Rev. D 94, 104015 (2016).

[66] T. Damour, Phys. Rev. D 97, 044038 (2018).

[67] D. Bini, T. Damour, and A. Geralico, Phys. Rev. Lett. 123, 231104 (2019).

[68] T. Damour and A. Nagar, Lect. Notes Phys. 905, 273 (2016).

[69] J. M. Henn, J. Phys. A 48, 153001 (2015).

[70] J. Parra-Martinez, M. S. Ruf, and M. Zeng, J. High Energy Phys. 11 (2020) 023.

[71] D. Neill and I. Z. Rothstein, Nucl. Phys. B877, 177 (2013).

[72] D. A. Kosower, B. Maybee, and D. O'Connell, J. High Energy Phys. 02 (2019) 137.

[73] B. Maybee, D. O'Connell, and J. Vines, J. High Energy Phys. 12 (2019) 156.

[74] C. Galley and R. A. Porto, J. High Energy Phys. 11 (2013) 096.

[75] B. R. Holstein and A. Ross, arXiv:0802.0716.

[76] N. Bjerrum-Bohr, J. F. Donoghue, and P. Vanhove, J. High Energy Phys. 02 (2014) 111.

[77] A. Guevara, J. High Energy Phys. 04 (2019) 033.

[78] M.-Z. Chung, Y.-T. Huang, J.-W. Kim, and S. Lee, J. High Energy Phys. 04 (2019) 156.

[79] A. Guevara, A. Ochirov, and J. Vines, J. High Energy Phys. 09 (2019) 056.

[80] W. D. Goldberger and A. K. Ridgway, Phys. Rev. D 97, 085019 (2018).

[81] S. Caron-Huot and Z. Zahraee, J. High Energy Phys. 07 (2019) 179.

[82] N. E. J. Bjerrum-Bohr, P. H. Damgaard, G. Festuccia, L. Plante, and P. Vanhove, Phys. Rev. Lett. 121, 171601 (2018).

[83] A. Cristofoli, N. E. J. Bjerrum-Bohr, P. H. Damgaard, and P. Vanhove, Phys. Rev. D 100, 084040 (2019).

[84] N. E. J. Bjerrum-Bohr, A. Cristofoli, and P. H. Damgaard, J. High Energy Phys. 08 (2020) 038.

[85] M.-Z. Chung, Y.-T. Huang, and J.-W. Kim, J. High Energy Phys. 09 (2020) 074.

[86] Y. F. Bautista and A. Guevara, arXiv:1903.12419.

[87] Y. F. Bautista and A. Guevara, arXiv:1908.11349.

[88] A. Koemans Collado, P. Di Vecchia, and R. Russo, Phys. Rev. D 100, 066028 (2019).

[89] A. Brandhuber and G. Travaglini, J. High Energy Phys. 01 (2020) 010.

[90] H. Johansson and A. Ochirov, J. High Energy Phys. 09 (2019) 040.
[91] R. Aoude, K. Haddad, and A. Helset, J. High Energy Phys. 05 (2020) 051.

[92] A. Cristofoli, P. H. Damgaard, P. Di Vecchia, and C. Heissenberg, J. High Energy Phys. 07 (2020) 122.

[93] Z. Bern, H. Ita, J. Parra-Martinez, and M. S. Ruf, Phys. Rev. Lett. 125, 031601 (2020).

[94] C. Cheung and M. P. Solon, J. High Energy Phys. 06 (2020) 144.

[95] C. Cheung and M. P. Solon, Phys. Rev. Lett. 125, 191601 (2020).

[96] M. Accettulli Huber, A. Brandhuber, S. De Angelis, and G. Travaglini, Phys. Rev. D 102, 046014 (2020).

[97] B. Grinstein, in Proceedings of the Workshop on HighEnergy Phenomenology (1991), p. 0161, https://doi.org/10 $.1142 / 1597$.

[98] G. Kälin, Z. Liu, and R. A. Porto (to be published).

[99] M. Beneke and V. A. Smirnov, Nucl. Phys. B522, 321 (1998).

[100] K. Chetyrkin and F. Tkachov, Nucl. Phys. B192, 159 (1981).

[101] F. Tkachov, Phys. Lett. B 100, 65 (1981).

[102] A. Smirnov and F. Chuharev, arXiv:1901.07808.

[103] R. Lee, arXiv:1212.2685.

[104] J. M. Henn, Phys. Rev. Lett. 110, 251601 (2013).

[105] M. Prausa, Comput. Phys. Commun. 219, 361 (2017).

[106] V. A. Smirnov, Analytic Tools for Feynman Integrals (Springer, New York, 2012).

[107] R. A. Porto, Phys. Rev. D 96, 024063 (2017).

[108] L. Barack and A. Pound, Rep. Prog. Phys. 82, 016904 (2019).

[109] A. Pound, B. Wardell, N. Warburton, and J. Miller, Phys. Rev. Lett. 124, 021101 (2020).

[110] D. Bini, T. Damour, and A. Geralico, Phys. Rev. D 102, 024062 (2020).

[111] J. Vines, J. Steinhoff, and A. Buonanno, Phys. Rev. D 99, 064054 (2019).

[112] N. Siemonsen and J. Vines, Phys. Rev. D 101, 064066 (2020).

[113] D. Bini, T. Damour, and A. Geralico, Phys. Rev. D 101, 044039 (2020).

[114] G. Kälin, Z. Liu, and R. A. Porto, Phys. Rev. D 102, 124025 (2020). 